\title{
ACTUALIZACIÓN DEL CONOCIMIENTO DE LA DISTRIBUCIÓN DE LA SUPERFAMILIA VITRINOIDEA FITZINGER, 1833 (GASTROPODA, PULMONATA) EN EL OESTE DE GALICIA
}

\author{
P. Ondina (*), J. Hermida (*) y T. Rodríguez (*)
}

\begin{abstract}
RESUMEN
En este trabajo se realiza un estudio faunístico de las especies de la Superfamilia Vitrinoidea Fitzinger, 1833, encontradas en el oeste de Galicia (provincias de A Coruña y Pontevedra). Para cada especie se incluyen citas previas y localidades de captura, así como sus respectivos mapas de distribución, en cuadrículas 10 x 10 Km U.T.M. Se constata la presencia de Aegopinella pura (Alder, 1830) en el área de estudio y se cita por primera vez Oxychilus glaber (Rossmässler, 1835) en la provincia de Pontevedra

Palabras Clave: Gastropoda, Pulmonata, Vitrinoidea, distribución, Galicia.
\end{abstract}

\begin{abstract}
An actualitation to the knowledge of the distribution of the Superfamily Vitrinoidea Fitzinger, 1833 (Gastropoda, Pulmonata) in the west of Galicia

A faunistic study of species of Superfamily Vitrinoidea Fitzinger, 1833, in the west of Galicia (A Coruña and Pontevedra provinces) has been realized. For each species the previous cite and the localities where the species have been found, are included, as well as a map showing the distribution of each species, using U.T.M. 10 x $10 \mathrm{Km}$ system. We corroborate the presence of Aegopinella pura (Alder, 1830) in the study area and Oxychilus glaber (Rossmässler, 1835) is recorded for first time from Pontevedra province.
\end{abstract}

Key words: Gastropoda, Pulmonata, Vitrinoidea, distribution, Galicia.

\section{Introducción}

Los primeros datos sobre la malacofauna terrestre en Galicia datan del siglo pasado, con los estudios llevados a cabo por Graells (1846), Seoane (1866), Macho Velado $(1871,1878)$ e Hidalgo (1875), continuándose posteriormente con trabajos más específicos y en zonas más con- cretas, como los realizados en la meseta y costa lucense por Altimira (1969), en las dunas de la ría de Vigo por Sacchi (1979) y Sacchi \& Violani (1977), en O Courel por Outeiro (1988) o en dos localidades de la provincia de A Coruña por Riballo (1990). Únicamente Castillejo (1981) aborda de forma extensa un estudio que cubre gran parte del territorio gallego, y en el que men-

* Departamento de Bioloxía Animal. Facultade de Bioloxía. Universidade de Santiago de Compostela. 15706 Santiago de Compostela. A Coruña, España. 
ciona un importante número de especies cuya presencia se desconocía hasta ese momento.

Aun así, muchas de las especies de gasterópodos terrestres citadas, tienen una distribución incierta e irregular, especialmente aquellas que por su pequeña talla necesitan técnicas de muestreo específicas (Coney et al., 1982; Bishop, 1977). Un claro ejemplo de esta situación lo presenta la Superfamilia Vitrinoidea de la que existe un único trabajo específico realizado por Castillejo (1985) que, aunque posee el mérito de darnos a conocer por primera vez en Galicia muchas de las especies que describe, las citas que aporta son escasas y dispersas, presentándose como una superfamilia de poca relevancia.

En el presente estudio se pretende dar a conocer la distribución de las especies de la Superfamilia Vitrinoidea en el oeste de Galicia (A Coruña y Pontevedra), utilizando un método de muestreo más específico y minucioso, que amplía notablemente el número de citas y contribuye a dar mayor coherencia a su distribución geográfica.

El material examinado se halla depositado en la colección del Departamento de Bioloxía Animal de la Facultade de Bioloxía de la Universidade de Santiago.

\section{Material y Métodos}

Durante un período de tres años se recolectó abundante material malacológico procedente de 176 localidades de las provincias de A Coruña y Pontevedra (Fig. 1a). Para su captura se examinaron detenidamente distintos tipos de hábitats (prados, bosques, puentes, muros, troncos, bajo piedras, basureros, etc.) tanto de día como de noche. Se recogieron, además, muestras de suelo y hojarasca de distintos biotopos, que posteriormente se lavaron y tamizaron para separar los ejemplares de pequeña talla.

Todos los ejemplares, tanto los procedentes de la recolección manual como los recogidos tras el lavado, se sometieron al proceso habitual de anoxia por inmersión en agua, con el fin de que muriesen relajados y facilitar de este modo la disección. Posteriormente se conservaron en alcohol de $70^{\circ}$.
A partir de los datos obtenidos se han elaborado los mapas de distribución en cuadrículas de 10x10 Km U.T.M. (Figs. 1b-c, 2a-i). En ellos se figuran las localidades aportadas en este trabajo (representadas por un punto negro) y las procedentes de la bibliografía (representadas por una estrella). Aquellas localidades en las que únicamente se han encontrado conchas vacías de una determinada especie se diferencian con una circunferencia. El listado de localidades con su correspondiente U.T.M. y código se exponen en la tabla I.

\section{Resultados}

Se han identificado un total de 6.979 ejemplares pertenecientes a 11 especies. Para cada una de ellas se listan, por orden cronólogico de publicación, aquellos autores que han citado la especie previamente a nosotros en el área de estudio, el material estudiado, incluyendo en este último el código de la localidad y el número de ejemplares, y el código de las localidades donde únicamente se han encontrado conchas vacías.

Familia VITRINIDAE Fitzinger, 1833

Vitrina (Vitrina) pellucida (O.F. Müller, 1774)

(Fig. 1b)

Citas previas: Sacchi \& Violani (1977); Castillejo (1985); Altonaga (1988a); Riballo (1990).

Material examinado (38 ejemplares): 10: 1. 17:

3. 27: 1. 34: 1. 35: 3. 36: 16. 83: 2. 86: 2. 94: 1.95:

1. 109: 1. 110: 2. 119: 2. 161: 2 .

Localidades con conchas vacías: 13.

Familia ZONITIDAE Mörch, 1864

Vitrea (Vitrea) contracta (Westerlund, 1871) (Fig. 1c)

Citas previas: Castillejo (1985); Otero-Schmitt \& Trigo-Trigo (1989); Riballo (1990).

Material examinado (391 ejemplares): 1: 1. 2: 1.

3: $1.4: 2.5: 2.6: 3.8: 3.9: 2.11: 6.12: 4.14: 19.16$ :

2. 17: 2. 19: 3. 20: 8. 24: 6. 25: 4. 26: 2. 27: 14. 28:

10. 29: 1. 30: 13. 31: 3. 32: 3. 35: 5. 36: 2. 37: 3. 38: 


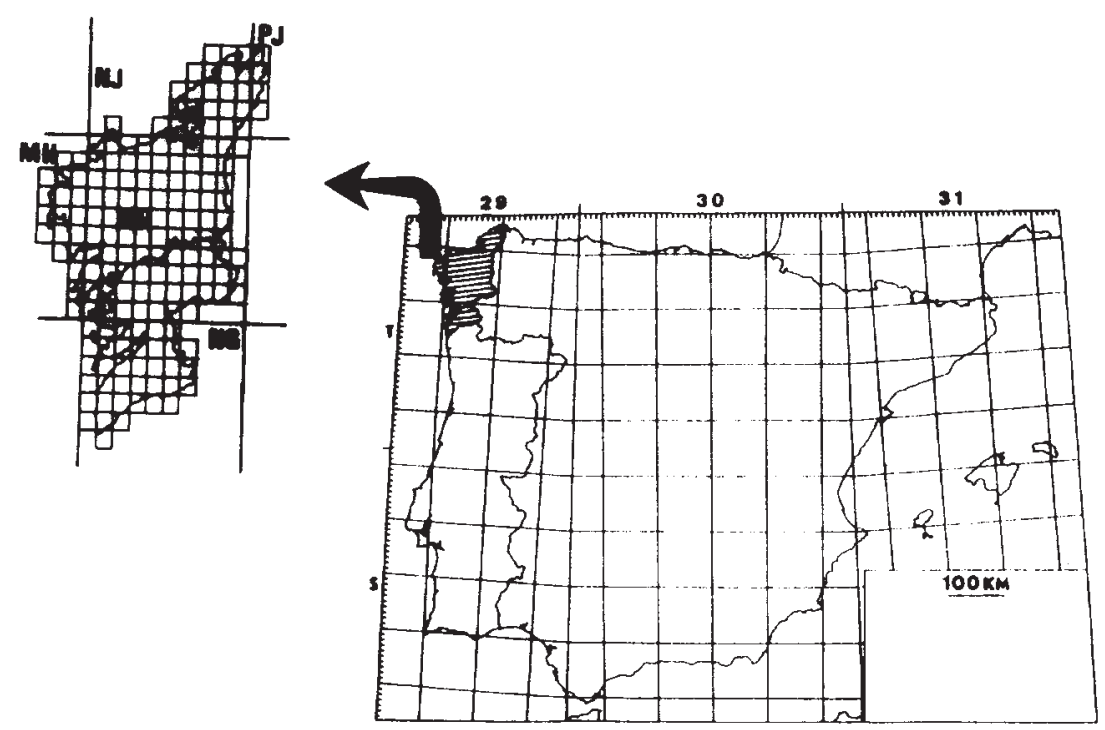

a
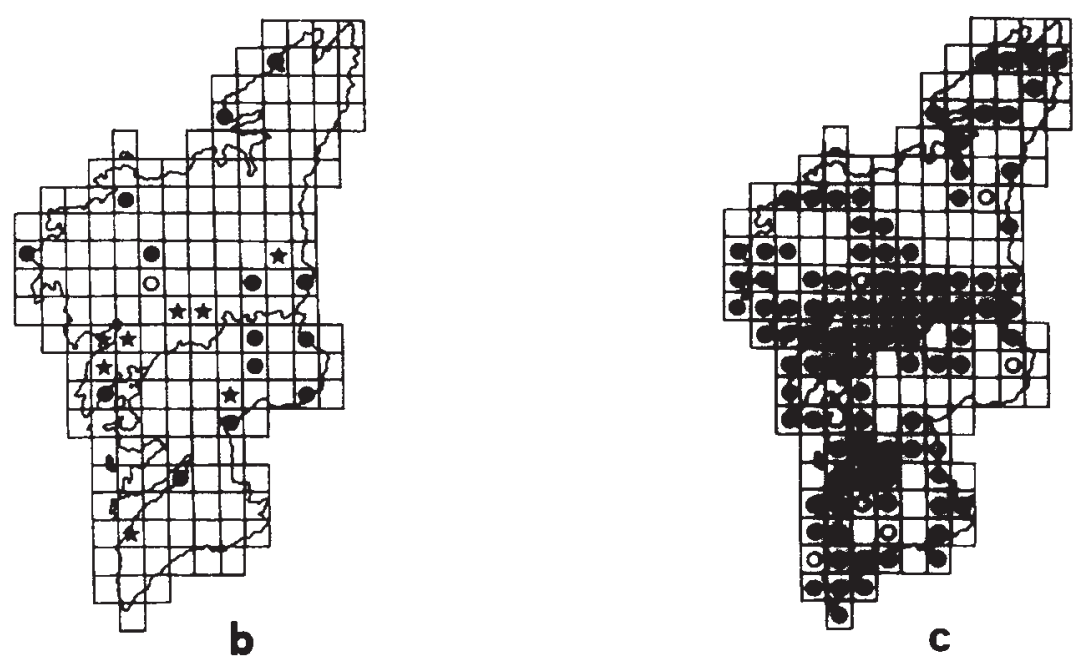

Fig. 1.- a: Area de estudio en la Península Ibérica. Mapas de distribución, b: V. pellucida, c: V. contracta.

Fig. 1.- a: The study area in the Iberian Peninsula. Distribution maps, b: V. pellucida, c: V. contracta,

1.39: 2. 40: 3. 42: 2. 43: 8. 46: 2. 47: 2. 49: 2. 50: 2 . 117: 5. 118: 3. 119: 3. 124: 1. 130: 2. 136: 2. 141: 2 . 52: $2.54: 2.55: 1.56: 1.58: 4.59: 7.60: 1.67: 3.69:$ 1.70: $10.74: 5.76: 2.77: 6.78: 3.79: 1.81: 11.82:$ 1.83: 6. 84: $1.85: 20.86: 3.88: 11.89: 3.93: 5.94:$ 17.95: 2.96: 1.99: 28. 100: 5. 102: 2. 103: 1. 104: 1 . 106: 2. 107: 1. 110: 3. 112: 1. 113: 1. 114: 19. 115: 1 .

152: 1. 156: 18. 157: 2. 158: 3. 160: 2. 162: 4.

Localidades con conchas vacías: 7. 13. 18. 21. 22. 41. 45. 57. 121. 148 . 

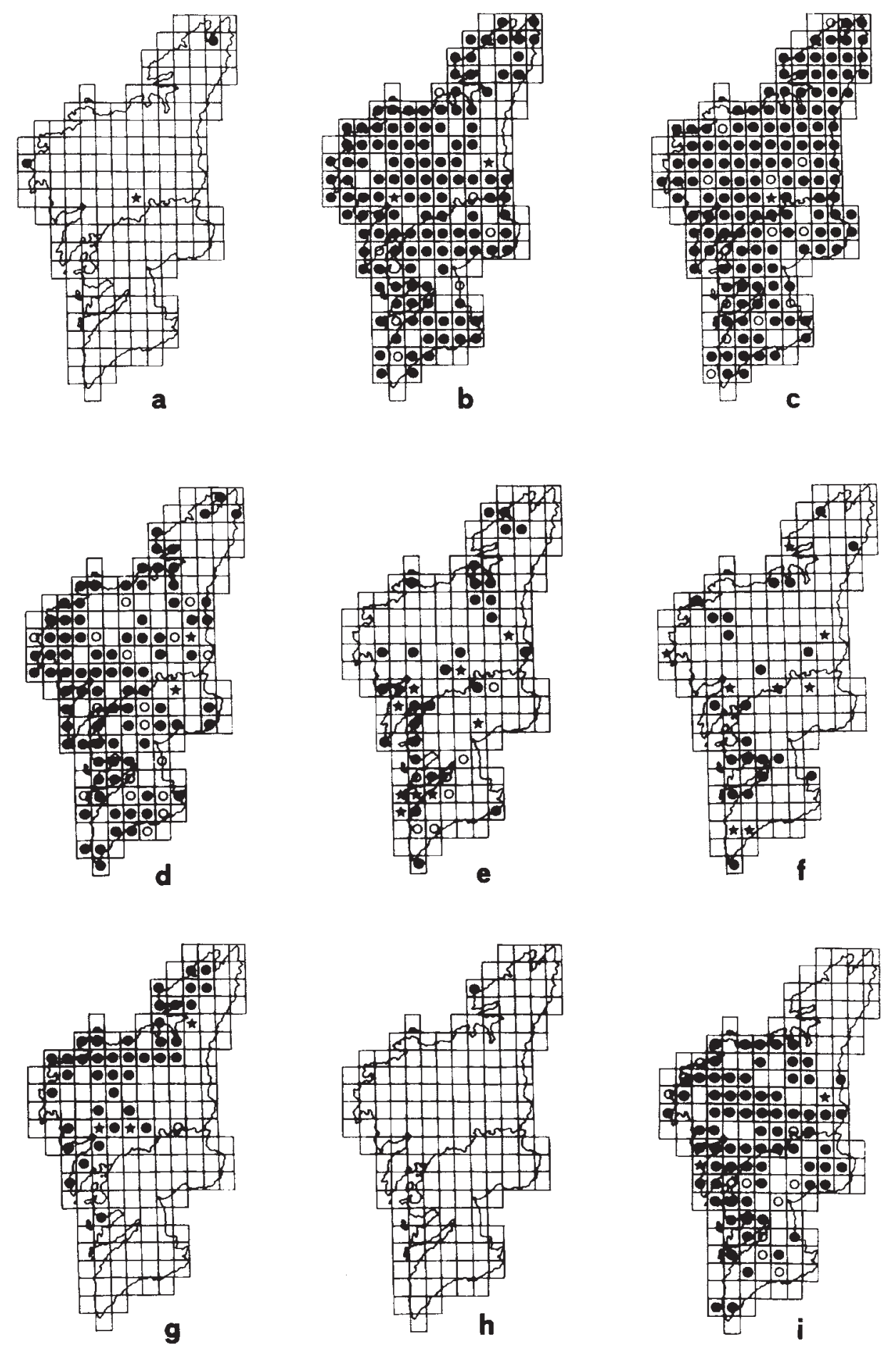

Fig. 2.- Mapas de distribución, a: A. pura, b: A. nitidula, c: N. hammonis, d: O. alliarius, e: O. cellarius, f: O. draparnaudi, g: O. glaber, h: Z. nitidus, i: Z. excavatus.

Fig. 2.- Distribution maps, a: A.pura, b: A.nitidula, c: N. hammonis, d: O. alliarius, e : O. cellarius, f: O.draparnaudi, g: $O$. glaber, h: Z. nitidus, i: Z. excavatus. 
Aegopinella pura (Alder, 1830) (Fig. 2a)

Citas previas: Riballo (1990)

Material examinado (4 ejemplares): 83: 3. 93: 1.

Aegopinella nitidula (Draparnaud, 1805)

(Fig. 2b)

Citas previas: Castillejo (1985); Altonaga (1988a); Riballo (1990).

Material examinado (1.168 ejemplares): 1: 2.3 : 5. 5: 10. 6: 9. 8: 4. 9: 4. 11: 2. 12: 7. 13: 5. 14: 5 . 15: 5. 17: 1. 18: 2. 19: 5. 21: 4. 22: 1. 24: 9. $26: 39$. 27: 44. 28: 4. 29: 19. 30: 9. 31: 10. 32: 2. 33: 5. 34: 1. 35: 13. 36: 6. 37: 14. 38: 3. 39: 3. 40: 4. 41: 4. 43: 7. 44: 10. 45: 13. 47: 11. 48: 1. 49: 15. 50: 15. 51: 8. 52: 13. 54: 4. 56: 17. 57: 13. 58: 12. 59: 35. 60: 163. 63: 1. 66: 3. 67: 25. 69: 1. 70: 1. 71: 2. 73: 2. 74: 2. 76: 25. 77: 41. 79: 2. 80: 1. 81: 10. 82: 4. 83: 7. 84: 1. 85: 3. 86: 2. 87: 10. 88: 10. 89: 1. 92: 1. 93: 5. 94: 2. 95: 16. 97: 18. 98: 2. 99: 4. 100: 4. 101: 3. 102: 1. 103: 1. 104: 3. 108: 5. 109: 8. 110: 2. 111: 11. 112: 18. 113: 15. 114: 1. 115: 6. 117: 40. 118: 1. 119: 15 . 122: 7. 124: 2. 126: 3. 128: 37.129 : 4. 130: 5. 131: 20. 132: 9. 133: 1. 134: 4. 135: 13. 136: 11. 141: 6. 145: 6. 146: 1. 147: 1. 149: 2. 151: 1. 152: 1. 153: 9. 154: 2. 155: 20. 156: 21. 157: 5. 159: 2. 160: 11. 162: 29. 167: 1. 169: 1 . 53.

Localidades con conchas vacías: 2. 4. 7. 10. 16.

\section{Nesovitrea hammonis (Ström, 1765) (Fig. 2c)}

Citas previas: Riedel (1979); Castillejo (1985); Riballo (1990).

Material examinado (3.258 ejemplares): 1: 7. 2: 2. 4: 26. 5: 9. 6: 9. 7: 1. 8: 9. 9: 1. 10: 10. 11: 22. 13: 3. 14: 6. 15: 2. 18: 3. 19: 1. 20: 9. 21: 7. $23: 11$. 25: 17.27: 15.29: 45.31: 8.33: 13.34: 1.36: 1.37: 6. 38: 1. 39: 23. 41: 11. 43: 30. 44: 43. 45: 2. 46: 2 . 47: 72. 49: 31. 50: 2. 51: 48. 52: 4.53: 5. 54: 10.55: 2. 56: 2. 57: 123. 58: 24. 59: 3. 61: 1. 62: 2. 64: 2 . 65: 2. 66: 8. 68: 5. 69: 5. 70: 1.71: 1.72: 16.74: 8 . 75: 6. 76: 16. 79: 4. 80: 15. 81: 144. 82: 3. 84: 73. 85: 4.87: 59. 88: 99. 89: 6. 90: 6.91: 44.92: 34.93: 45. 94: 171.95: 42.96: 69.97: 171.98: 202.99: 8. 100: 9. 101: 29. 102: 154. 103: 48. 104: 84. 105: 15.
106: 9. 107: 13. 108: 7. 109: 2. 110: 6. 111: 2.112 : 32. 115: 16. 116: 113. 117: 6. 118: 49. 119: 7. 120: 5. 121: 18. 122: 45. 123: 39. 124: 35. 125: 4. 126: 135. 127: 2. 128: 19. 129: 4. 130: 20. 131: 51. 132: 44. 134: 9. 135: 58. 136: 68. 137: 12. 139: 1. 140: 14. 141: 10. 142: 21. 143: 1. 144: 2. 145: 19. 147: 3. 148: 5. 149: 2. 150: 4. 151: 1. 152: 13. 154: 21. 156: 1. 159: 1. 160: 1. 163: 1. 164: 4. 165: 8. 166: 2.

Localidades con conchas vacías: 3. 17. 22. 23. 26. 28. 32. 48. 67. 77. 86. 138. 146. 157.

Oxychilus (Ortizius) alliarius (Miller, 1822) (Fig. 2d)

Citas previas: Castillejo (1985); Altonaga (1988a); Riballo (1990).

Material examinado (454 ejemplares): 1: 4. 4: 3. 5: 3. 6: 9. 11: 1. 13: 2. 14: 2. 18: 2. 21: 2. 25: 27. 28: 9. 29: 25. 30: 25. 31: 2. 33: 1. 34: 7. 37: 3. 39: 8. 40: 2. 42: 20. 43: 6. 44: 6. 47: 8. 48: 2. 49: 14 . 50: 1. 51: 1. 52: 2. 56: 2. 57: 1.58: 1. 61: 3. 63: 1 . 67: 5. 68: 7. 69: 28. 71: 2.73: 6. 74: 3. 75: $1.77: 5$. 78: $23.79: 15.81: 4.82: 2.84: 1.85: 4.88: 1.89$ : 1. 90: 1. 94: 4. 95: 2. 99: 3. 100: 3. 101: 3. 102: 4. 103: 4. 107: 2. 112: 1. 113: 3. 114: 11. 115: 4. 116: 6. 117: 2. 125: 1. 126: 1. 128: 10. 129: 1. 131: 3 . 133: 3. 135: 2. 136: 3. 137: 1. 140: 1. 141: 9. 148: 3. 149: 12. 150: 2. 153: 2. 154: 2. 155: 20. 156: 4. 160: 2. 165: 6 .

Localidades con conchas vacías: 3. 7. 8. 15. 23. 24. 27. 32. 59. 60. 70.80.83. 87. 110. 121 . 138.

Oxychilus (Oxychilus) cellarius (Müller, 1774) (Fig. 2e)

Citas previas: Macho Velado (1871); Hidalgo (1875); Hidalgo (1890); Sacchi \& Violani (1977); Castillejo (1981, 1985); Otero-Schmitt \& TrigoTrigo (1989); Riballo (1990).

Material examinado (91 ejemplares): 6: 2. 12: 1 26: 7. 37: 1. 42: 3. 48: 4. 57: 1. 58: 12. 61: 5. 64: 10. 69: 4. 70: 1. 74: 5. 79: 4. 94: 1. 97: 1. 100: 2 . 108: 1. 110: 1. 123: 12. 127: 1. 128: 3. 133: 1. 137: 2. 141: 2. 143: 2. 159: 1. 169:1

Localidades con conchas vacías: 2.3.27. 28.35. 43. 46. 
Oxychilus (Oxychilus) draparnaudi (Beck, 1837) (Fig. 2f)

Citas previas: Sacchi \& Violani (1977); Castillejo (1985).

Material examinado (78 ejemplares): 4: 2. 5: 1 . 6: 15. 27: 1. 29: 5. 37: 2. 38: 1. 42: 1. 45: 4. 46: 1 . 47: 1. 79: 1. 80: 4. 85: 12.92: 1. 94: 6.97: 8. 109: 1. 132: 1. 149: 2. 154: 7. 168: 1 .

Oxychilus (Morlina) glaber (Rossmässler, 1835) (Fig. 2g)

Citas previas: Castillejo (1985); Altonaga (1988b); Otero-Schmitt \& Trigo-Trigo (1989)

Material examinado (291 ejemplares): 6: 4. 38: 2. 39: 4. 40: 5. 49: 5. 50: 2. 57: 1.58: 7. 59: 33.85: 1.86: 1. 87: 1. 94: 6. 95: 2. 96: 3. 97: 1. 100: 16 . 101: 11. 102: 30. 111: 1. 113: 2. 115: 1. 117: 16 . 118: 2. 119: 5. 123: 8. 126: 40.127: 11. 128: 7. 130: 28. 131: 14. 132: 3. 133: 1. 134: 5. 135: 7. 141: 1 . 143: 2. 152: 1. 153: 1 .

Localidades con conchas vacías: $\mathbf{1 6}$.

Zonitoides (Zonitoides) nitidus (Müller, 1774) (Fig. 2h)

Citas previas: Ondina et al. (1995): 126.

Material examinado (1 ejemplar): 126: 1.

Zonitoides (Zonitoides) excavatus (Alder, 1830) (Fig. 2i)

Citas previas: Riedel (1979); Castillejo (1985); Riballo (1990).

Material examinado (1.205 ejemplares): 4: 21. 5: 16. 6: 9. 10: 28. 11: 24. 12: 2. 13: 10. 14: 1. 16: 1. 17: 1. 18: 9. 20: 14. 22: 8. 25: 1. 29: 74. 30: 21. 33: 9. 37: 31.38: 2. 40: 8. 44: 1. 47: 1. 49: 1.50: 3. 51: 3. 52: 6. 53: 2. 57: 44. 58: 54. 59: 3. 61: 6. 64: 1. 65: 14. 66: $1.70: 2.71: 61.74: 33.75: 2.77: 1$. 79: 22. 80: 2. 81: 54. 87: 3. 88: 83. 89: 1.97: 57.98: 48. 99: 18. 107: 9. 108: 13. 109: 1. 110: 2. 112: 26. 113: 8. 115: 6. 116: 9. 117: 42. 119: 20. 127: 3.131: 27. 132: 25. 135: 9. 136: 75. 141: 48. 149: 11. 151: 21. 152: 15. 153: 3. 154: 14. 157: 1.
Localidades con conchas vacías: 3. 9. 27. 31. 48. 55. 63. 73. 83. 85. 155.

\section{Discusión}

La mayoría de las especies encontradas presentan una distribución europea $(O$.glaber, $O$. draparnaudi, $O$. alliarius, $O$. cellarius, A. nitidula y $A$. pura), otras poseen una rango de distribución más amplio, holártico (Z. nitidus y V. pellucida) o paleártico ( $V$. contracta y $N$. hammonis). La distribución más restringida la posee $Z$. excavatus, que ocupa el área atlántica de Europa.

Las especies de mayor presencia en el área de estudio fueron $N$. hammonis (que aparece en el $75,15 \%$ del total de las cuadrículas examinadas), $A$. nitidula $(68,36 \%)$ y $V$. contracta $(50,28 \%)$. Así mismo las que aparecen en un porcentaje más bajo son Z. nitidus $(0,56 \%)$ y A. pura $(1,12 \%)$. El motivo de la escasa presencia de estas dos especies parece estar ligado a factores ambientales, aunque con diferentes connotaciones.

Las referencias ecológicas que poseemos de Z.nitidus la presentan como una especie intimamente ligada a los cursos de agua (Kerney \& Cameron, 1979; Altonaga 1988a, 1990), que se encuentra en zonas pantanosas y márgenes de ríos y lagos, medio que generalmente no se examina de forma minuciosa en los estudios malacológicos terrestres. Es probablemente este hecho el que le da a su distribución en la Península cierto carácter fragmentado, encontrándose más representada en el norte peninsular y de forma dispersa y aislada en el resto, lo que hace difícil su interpretación.

La única cita de esta especie en Galicia corresponde a un prado de regadío con un alto grado de humedad, pero por lo expuesto anteriormente creemos muy probable que se encuentre mejor representada en los márgenes de los ríos gallegos.

En lo que respecta a A. pura se había mencionado hasta el momento en una única localidad de la zona de estudio (Riballo, 1990), siendo citada por primera vez en Galicia y de forma abundante por Outeiro (1988) en la Sierra do Courel (Lugo). Esta especie es considerada por la mayoría de los autores como característica de bosques (Kerney \& 
Tabla I. - Listado de las localidades, código y U.T.M. de 10 x 10 km.

Table I. - List of localities, register number and U.T.M. 10 x $10 \mathrm{~km}$.

\begin{tabular}{|c|c|c|c|c|c|}
\hline Código & Localidad/ Ayuntamiento & UTM & Código & Localidad/Ayuntamiento & UTM \\
\hline 1 & Caldelas de Tui, Tui & 29TNG35 & 56 & Rellas, Silleda & 29TNH52 \\
\hline 2 & Cristelos, Tomiño & 29TNG15 & 57 & Louro, Muros & 29TMH93 \\
\hline 3 & Louredo, Mos & 29TNG37 & 58 & Ordoeste, А Ваña & 29TNH15 \\
\hline 4 & Coruxo, Vigo & 29TNG17 & 59 & A Peregrina, Santiago & 29TNH35 \\
\hline 5 & Pintos, Pontevedra & 29TNG39 & 60 & Illas Cíes, Vigo & 29TNG07 \\
\hline 6 & Dorrón, Sanxenxo & 29TNG19 & 61 & Barrantes, Ribadumia & 29TNH10 \\
\hline 7 & A Xesta, A Lama & 29TNG59 & 62 & Fontáns, Barro & 29TNH30 \\
\hline 8 & O Covelo, O Covelo & 29TNG57 & 63 & Forcarei, Forcarei & 29TNH51 \\
\hline 9 & Caldas de Reis, Caldas de Reis & 29TNH21 & 64 & Lamela, Silleda & 29TNH53 \\
\hline 10 & Sobrido, Ribeira & 29TNH01 & 65 & Muimenta, Lalín & 29TNH73 \\
\hline 11 & Noal, Porto do Son & 29TNH03 & 66 & A Xesta, Lalín & 29TNH71 \\
\hline 12 & Sionlla, Santiago & 29TNH45 & 67 & Baroña, Porto do Son & 29TМН92 \\
\hline 13 & Negreira, Negreira & 29TNH25 & 68 & Pontenafonso, Noia & 29TNH14 \\
\hline 14 & Ponte Sarandón, Vedra & 29TNH43 & 69 & Carreira, Ribeira & 29TMH90 \\
\hline 15 & Arca, A Estrada & 29TNH41 & 70 & Bures, Catoira & 29TNH12 \\
\hline 16 & Ferreiros, Vila de Cruces & 29TNH64 & 71 & Sta Mariña de Barcala, A Estrada & 29TNH32 \\
\hline 17 & Prado, Lalín & 29TNH62 & 72 & Oitavén, Fornelos de Montes & 29TNG48 \\
\hline 18 & Santa María, Rodeiro & 29TNH82 & 73 & Ponteareas, Ponteareas & 29TNG46 \\
\hline 19 & Amance, Golada & 29TNH84 & 74 & Moaña, Moaña & 29TNG28 \\
\hline 20 & Herbón, Padrón & 29TNH23 & 75 & Mosende, Porriño & 29TNG26 \\
\hline 21 & Budiño, Porriño & 29TNG36 & 76 & Tomiño, Tomiño & 29TNG24 \\
\hline 22 & Piñeiro, O Covelo & 29TNG58 & 77 & Chavella, Oia & 29TNG04 \\
\hline 23 & Salvaterra, Salvaterra do Miño & 29TNG45 & 78 & Cabo Silleiro, Baiona & 29TNG06 \\
\hline 24 & Arbo, Arbo & 29TNG56 & 79 & Santiago, Santiago & 29TNH34 \\
\hline 25 & Loureza, Oia & 29TNG14 & 80 & Esmorode, Santa Comba & 29TNH16 \\
\hline 26 & Baredo, Baiona & 29TNG16 & 81 & Serra de Outes, Serra de Outes & 29TNH04 \\
\hline 27 & Amoedo, Pazos de Borbén & 29TNG38 & 82 & Quilmas, Carnota & 29TMH84 \\
\hline 28 & Magdalena, Cangas & 29TNG18 & 83 & Vilela, Muxía & 29TMH76 \\
\hline 29 & Leiro, Ribadumia & 29TNH20 & 84 & Berdoias, Vimianzo & 29TMH96 \\
\hline 30 & S. Vicente do Grove, O Grove & 29TNHOO & 85 & Nande, Laxe & 29TMH98 \\
\hline 31 & Serrapio, Cerdedo & 29TNH40 & 86 & As Tarandeiras, Coristanco & 29TNH18 \\
\hline 32 & Nigoi, A Estrada & 29TNH42 & 87 & Torás, Laracha & 29TNH38 \\
\hline 33 & Vilatuxe, Lalín & 29TNH61 & 88 & Rial, Trazo & 29TNH36 \\
\hline 34 & Lagoa, Dozón & 29TNH81 & 89 & Zas de Reis, Melide & 29TNH75 \\
\hline 35 & Barrio, Vila de Cruces & 29TNH63 & 90 & Padreiro, Curtis & 29TNH77 \\
\hline 36 & Ventosa, Golada & 29TNH83 & 91 & Xestal, Irixoa & 29TNH79 \\
\hline 37 & Catoira, Catoira & 29TNH22 & 92 & Saa, As Pontes & 29TNJ91 \\
\hline 38 & Boiro, Boiro & 29TNH02 & 93 & Freires, Ortigueira & 29TNJ93 \\
\hline 39 & Lapido, Ames & 29TNH24 & 94 & Regoa, Cedeira & 29TNJ73 \\
\hline 40 & Sergude, Boqueixón & 29TNH44 & 95 & Esmelle, Ferrol & 29TNJ51 \\
\hline 41 & Costa de Mougás, Oia & 29TNG05 & 96 & Bouzarredonda, Neda & 29TNJ71 \\
\hline 42 & Camposancos, A Guarda & 29TNG13 & 97 & Sigrás, Cambre & 29TNH59 \\
\hline 43 & Sobrada, Tomiño & 29TNG25 & 98 & Abellá, Frades & 29TNH57 \\
\hline 44 & Mondariz, Mondariz & 29TNG47 & 99 & Pedrouzo, O Pino & 29TNH55 \\
\hline 45 & Lavadores, Vigo & 29TNG27 & 100 & Viñas, Paderne & 29TNH69 \\
\hline 46 & Pontecaldelas, Pontecaldelas & 29TNG49 & 101 & Neda, Neda & 29TNJ61 \\
\hline 47 & Postemirón, Vilaboa & 29TNG29 & 102 & Ponte de Mera, Ortigueira & 29TNJ83 \\
\hline 48 & Triñáns, Boiro & 29TNH11 & 103 & Mañón, Mañón & 29TPJ03 \\
\hline 49 & Oleiros, Ribeira & 29ТМН91 & 104 & Recemel, Somozas & 29TNJ81 \\
\hline 50 & Portobravo, Lousame & 29TNH13 & 105 & Boliqueiras, As Pontes & 29TNJ90 \\
\hline 51 & César, Caldas de Reis & 29TNH31 & 106 & Salto do Conexo, Monfero & 29TNH89 \\
\hline 52 & Carcacía, Padrón & 29TNH33 & 107 & Monte do Arco, Curtis & 29TNH87 \\
\hline 53 & Carballeda, Lalín & 29TNH72 & 108 & Rexidoira, Cesuras & 29TNH67 \\
\hline 54 & Baíña, A Golada & 29TNH74 & 109 & Arzúa, Arzúa & 29TNH65 \\
\hline 55 & Quintás, Touro & 29TNH54 & 110 & Furelos, Melide & 29TNH85 \\
\hline
\end{tabular}


Tabla I.- Cont.

\begin{tabular}{|c|c|c|c|c|c|}
\hline Código & Localidad/ Ayuntamiento & UTM & Código & Localidad/Ayuntamiento & UTM \\
\hline 111 & Vila da Igreza, Cerceda & 29 TNH48 & 141 & Burricios, Oza dos Ríos & 29TNH68 \\
\hline 112 & Parada, Ordes & 29TNH46 & 142 & Faeira, As Pontes & 29TNJ80 \\
\hline 113 & Pedrafigueira, Carnota & 29ТМН94 & 143 & As Somozas, Somozas & 29TNJ82 \\
\hline 114 & Cabo Fisterra, Fisterra & 29TMH74 & 144 & Grañas, Mañón & 29TPJ02 \\
\hline 115 & Ozón, Muxía & 29TMH86 & 145 & Loiba, Ortigueira & 29TPJ04 \\
\hline 116 & Areosa, Vimianzo & 29TNH06 & 146 & Serra da Capelada, Ortigueira & 29TNJ84 \\
\hline 117 & Ponteceso, Ponteceso & 29TNH08 & 147 & Sedes, Narón & 29TNJ62 \\
\hline 118 & Cances, Carballo & 29TNH28 & 148 & Laraxe, Cabanas & 29TNJ60 \\
\hline 119 & Bembibre, Val do Dubra & 29TNH26 & 149 & Ledoño, Culleredo & 29TNH49 \\
\hline 120 & Sobrado dos Monxes, Sobrado & 29TNH76 & 150 & Leira, Ordes & 29TNH47 \\
\hline 121 & A Castellana, Aranga & 29TNH78 & 151 & Razo, Carballo & 29TNH29 \\
\hline 122 & A Capela, A Capela & 29TNJ70 & 152 & Canosa, Carballo & 29TNH27 \\
\hline 123 & San Sadurniño, San Sadurniño & 29TNJ72 & 153 & Corme, Ponteceso & 29TNH09 \\
\hline 124 & O Ermo, Ortigueira & 29TNJ92 & 154 & Baio, Zas & 29TNH07 \\
\hline 125 & Sismundi, Ortigueira & 29TNJ94 & 155 & Moraime, Muxía & 29TМH87 \\
\hline 126 & Raxón, Ferrol & 29TNJ52 & 156 & Lobelos, Cée & 29TMH85 \\
\hline 127 & Mabegondo, Abegondo & 29TNH58 & 157 & A Picota, Mazaricos & 29TNH05 \\
\hline 128 & Carnoedo, Sada & 29TNJ50 & 158 & Setados, As Neves & 29TNG55 \\
\hline 129 & Poulo, Ordes & 29TNH56 & 159 & Crecente, Crecente & 29TNG66 \\
\hline 130 & Río, Cerceda & 29TNH37 & 160 & Paredes, A Cañiza & 29TNG67 \\
\hline 131 & Noicela, Carballo & 29TNH39 & 161 & Pardesoa, Forcarei & 29TNH50 \\
\hline 132 & Agualada, Coristanco & 29TNH17 & 162 & Lira, Carnota & 29TМH83 \\
\hline 133 & Buño, Malpica & 29TNH19 & 163 & Couto, Rodeiro & 29TNH93 \\
\hline 134 & Santa Mariña, Camariñas & 29TMH88 & 164 & Vilafrío, Rodeiro & 29TNH92 \\
\hline 135 & Ponte do Porto, Camariñas & 29TMH97 & 165 & San Pedro de Visma, A Coruña & 29TNJ40 \\
\hline 136 & Fisterra, Fisterra & 29TMH75 & 166 & Sucadio, As Pontes & 29TPJ01 \\
\hline 137 & Logoso, Dumbría & 29TMH95 & 167 & Illa de Ons, Bueu & 29TNG09 \\
\hline 138 & Arceo, Boimorto & 29TNH66 & 168 & Vilaverde, Ribadavia & 29TNG68 \\
\hline 139 & Lagoa de Sobrado, Sobrado & 29TNH86 & 169 & Punta Frouxeira, Valdoviño & 29TNJ63 \\
\hline
\end{tabular}

Cameron, 1979; Giusti, 1971; Mordan, 1977). Así, Outeiro (1988) la encuentra con mayor frecuencia en los arbolados y asociada a suelos de textura gruesa, húmedos y con bajos valores de calcio y $\mathrm{pH}$. Con estas referencias resulta en cierta forma sorprendente que hayamos encontrado unicamente cuatro ejemplares de A. pura en un borde de río y un arbolado (pinar), ya que se han recogido muestras en un número elevado de bosques y que la mayor parte de los suelos del área de estudio se encuentran dentro de los valores descritos por Outeiro (1988) como óptimos para dicha especie. Todo ello, y dado el tipo de metodología exhaustiva que hemos utilizado, nos indica que, esta especie europea, posee una escasa representación en el área de estudio. Con las citas aportadas en este tra- bajo, además de constatar su presencia en Galicia, se amplía el límite occidental de su distribución conocida en la Península Ibérica.

El hallazgo de más de mil ejemplares de $Z$. excavatus tiene un interés especial ya que es la única especie atlántico-europea que, en la Península Ibérica, se encuentra limitada a Galicia. Este hecho podría deberse a que se trata de una especie restringida a suelos ácidos no calcáreos (Boycott, 1934; Kerney \& Cameron, 1979; Bishop, 1969), teoría que se ve reforzada por el alto número de citas aportadas en este estudio, donde la mayoría de los suelos se desarrollan sobre granito.

En la franja occidental gallega se encuentran todas las especies de la superfamilia Vitrinoidea descritas en Galicia, y citadas por Castillejo (1985), 
con la excepcion de Retinella incerta (Draparnaud, 1805). Esta especie, tras la cita de Castillejo (1981, 1985) en O Courel (Lugo) no ha vuelto a ser encontrada, ni en el exhaustivo estudio realizado por Outeiro (1988) en esta sierra. Esto nos ha llevado a visitar dicha zona con resultados negativos y a poner, por tanto, en duda su presencia en Galicia, pese a la certeza de su correcta identificación. Esta duda es compartida por el Dr. Castillejo (com. pers.) que nos confirmó que el material no fue recogido por él y que muy probablemente fue confundido con otras muestras procedentes de Navarra.

Queremos destacar que con este trabajo se amplía notablemente el área de distribución conocida de las distintas especies, lo que nos da una idea más real de su presencia en Galicia. Así, por ejemplo, se ha multiplicado por ocho las escasas citas conocidas hasta el momento de $V$. contracta, $A$. nitidula ha pasado de 10 a 113 citas y, para N. hammonis hemos aumentado el número de localidades en 124.

\section{Referencias}

Altimira, C., 1969. Notas Malacológicas. VIII Moluscos del Delta del Llobregat. IX Nuevas aportaciones y datos a la fauna malacológica catalana. XI Moluscos terrestres y de agua dulce recogidos en la provincia de Lugo y en Asturias. Pub. Inst. Biol. Apl., 46: 91-113.

Altonaga, K., 1988a. Estudio taxonómico y biogeográfico de las Familias Endodontidae, Euconulidae, Zonitidae y Vitrinidae (Gastropoda: Pulmonata: Stylommatophora) de la Península Ibérica, con especial referencia al País Vasco y zonas adyacentes. Tesis Doctoral (inédita). Universidad del País Vasco. 549 pp.

Altonaga, K., 1988b. Nuevos datos sobre Oxychilus (Morlina) glaber (Rossmässler, 1835) (Pulmonata: Stylommatophora: Zonitidae) en la Península Ibérica. Iberus, 8: 39-45.

Altonaga, K., 1990. Sobre Zonitoides nitidus (O. F. Müller, 1774) (Pulmonata, Stylommatophora, Zonitidae) en la Península Ibérica. Cuad. Invest. Biol., 16: 35-52.
Bishop, M.J., 1977. Approaches to the quantitative description of terrestrial mollusc populations and habitats. Malacologia , 16: 61-66.

Boyсотт, A.E., 1934. The habitat of land molusca in Britain. J. Ecol., 22: 1-38.

Castillejo, J., 1981. Los moluscos terrestres de Galicia (Subclase Pulmonata). Tesis Doctoral (inédita). Universidad de Santiago. 499 pp. + 16 lam.

Castillejo, J., 1985. Caracoles terrestres de Galicia, II. Familia Zonitidae (Gastropoda, Pulmonata). Iberus, 5: 63-81.

Coney, C., Wallace, A., Tarpley, J. \& Nagel, W., 1982. Ecological studies of land snails in the Hiwasse River Basin of Tenesse, U.S.A. Malacol. Rev., 15: 69-106.

GiUsTI, F., 1971. Notulae malacologicae XVI. I Molluschi terrestri e di acqua dolce viventi sul Massiccio dei Monti Reatini (Appennino Centrale). Lavori Soc. Ital. Biogeogr. (n.s.), 2: 423-576+7 lam.

GRAELls, M.P., 1846. Catálogo de los moluscos terrestres y de agua dulce observados en España y descripción y notas de algunas especies nuevas o poco conocidas del mismo país. $33 \mathrm{pp}$. Madrid.

Hidalgo, J.G., 1875. Catálogo iconográfico y descriptivo de los moluscos terrestres de España, Portugal y las Baleares. Madrid. Parte 1A: 224 pp., Parte 2A: 16 pp.

Hidalgo, J.G., 1890. Catálogo de los moluscos recogidos en Bayona de Galicia. Rev. Progr. Cienc. Exact. Fís. Nat., 21: 396-397.

KeRNey, M.P. \& CAMERON, R.A.D., 1979. A field guide to the land snails of Britain and Northwest Europe. Collins Sons and Co Ltd., Glasgow, $288 \mathrm{pp}$.

Macho Velado, J., 1871. Catálogo de los moluscos terrestres observados en Galicia. Hoj. Malacol. Madrid, 10-16.

Macho Velado, J., 1878. Moluscos de agua dulce de Galicia con observaciones sobre las especies y localidades. An. Soc. Esp. Hist. Nat., 7: 235248.

Mordan, P.B., 1977. Factors affecting the distribution and abundance of Aegopinella and 
Nesovitrea (Pulmonata: Zonitidae) at Monks wood Nature Reserve, Huntingdonshire. Biol. J. Linn. Soc., 9: 59-62.

Ondina, P., Hermida, J. \& Outeiro, A., 1995. Nuevas citas de cuatro gasterópodos terrestres para Galicia. Nova Acta Científ. Compost., 5: 215-224.

Otero-Schmitt, J. \& Trigo-Trigo, J., 1989. Moluscos de la ría de Muros. Thalassas, 7: 7990.

Outeiro, A., 1988. Gasterópodos de O Courel (Lugo). Universidad de Santiago. Tesis Doctoral (inédita). 626 pp.

Riballo, I., 1990. Gasterópodos terrestres de Rubio-Boqueixón y Cernán-Rois (La Coruña). Tesis Doctoral (inédita). Universidad de Santiago. 399 pp. 2 lam.

Riedel, A., 1979. Materialien zur Kenntnis der paläarktischen Zonitidae (Gastropoda). XIIXIV. Fragmenta Faunistica, XXV, 9: 115-125.
SACCHI, C.F., 1979. Note ecologique sulla malacofauna dell'Aragona Settentrionale (Spagna). Boll. Mus. Civ. Venezia, 30: 76-99.

Sacchi, C. \& Violani, C., 1977. Richerche ecologiche sulle elicide dunicole della Ría di Vigo (Spagna), (Gastropoda, Pulmonata), Natura, 68: 253-284.

SEOANe, V.L., 1866. Reseña de la Historia Natural de Galicia. Lugo. 66 p.
Recibido, el 28-VIII-1995

Aceptado, el 3-X-1996

Publicado, el 27-XII-1996 